\title{
Themenschwerpunkt Soziale Elternschaft
}

\author{
Thomas Rauschenbach • Heinz-Hermann Krüger • Ivo Züchner
}

Online publiziert: 4. April 2020

(C) Der/die Autor(en) 2020

„Pflege und Erziehung der Kinder sind das natürliche Recht der Eltern und die zuvörderst ihnen obliegende Pflicht", so lautet die Formulierung in Artikel 6 des Grundgesetzes. Diese naturrechtlich formulierte Verhältnis zwischen „Eltern“ und „Kindern“ beruht auf der impliziten Annahme, dass Elternschaft biologisch begründbar ist, dass Mütter und Väter mithin die leiblichen Eltern ihrer Kinder sind. Dieser menschheitsgeschichtliche Regelfall ist im Laufe der vergangenen Jahrzehnte jedoch immer häufiger durch andere, nicht-biologisch begründete Formen der Elternschaft ergänzt worden. Unstrittig zu beobachten ist, dass Eltern sich heutzutage häufiger trennen oder scheiden lassen, dass Mütter oder Väter mit ihren Kindern zeitweilig alleine leben und später wieder eine neue Partnerschaft eingehen.

Für alle Formate einer Elternschaft, die nicht auf einer biologischen, leiblichen Elternschaft beruhen, wird heutzutage in den Sozialwissenschaften zumeist der Begriff „,soziale Elternschaft“" verwendet. „Soziale Elternschaften“, die historisch keineswegs neu, jedoch heutzutage sehr viel häufiger anzutreffen sind, entstehen zum einen, wenn der neue Partner oder die neue Partnerin die Rolle eines Elternteils auch für jene Kinder in der neuen Partnerschaft übernimmt - von der emotionalen Zuwendung über finanzielle Verpflichtungen bis zu einer generellen Verantwortungsübernahme für das Aufwachsen dieser Kinder -, die biologisch nicht von dem neuen

Prof. Dr. T. Rauschenbach $(\bowtie)$

DJI - Deutsches Jugendinstitut, Nockherstr. 2, 81541 München, Deutschland

E-Mail: rauschenbach@dji.de

Prof. Dr. H.-H. Krüger

Martin-Luther-Universität Halle/Wittenberg., Franckeplatz 1, 06099 Halle/Saale, Deutschland

E-Mail: heinz-hermann.krueger@paedagogik.uni-halle.de

Prof. Dr. I. Züchner

Phillipps Universität Marburg, Wilhelm-Röpke-Straße 6, 35032 Marburg, Deutschland

E-Mail: zuechner@staff.uni-marburg.de 
Elternteil abstammen. Sie sind zum anderen aber auch dann gegeben, wenn Kinder mithilfe der Reproduktionsmedizin gezeugt werden, oder Kinder in homosexuellen Partnerschaften aufwachsen. Und als Formen sozialer Elternschaft gelten schließlich auch jene Kindschaftsverhältnisse, bei denen Kinder adoptiert werden oder die im Rahmen einer „Hilfe zur Erziehung“ nach $§ \$ 27 \mathrm{ff}$ SGB VIII zeitweilig in einer Pflegefamilie, in einer Wohngruppe oder einem Heim leben.

Auch wenn manche dieser Formen zahlenmäßig weiterhin nur einen kleinen Anteil der Eltern-Kind-Verhältnisse ausmachen, so stellen sie unter rechtlichen Gesichtspunkten eine mindestens ebenso große Herausforderung dar wie unter erziehungs - und sozialwissenschaftlichen Gesichtspunkten, fällt in all diesen Fällen doch die naturrechtlich-biologische Begründung für Elternschaft weg. Die damit verbundenen Fragen, die sich in modernen Eltern-Kind-Verantwortungsgemeinschaften in einer immer größer werdenden Zahl an Fällen stellt, stehen im Mittelpunkt dieses Themenschwerpunktes.

In dem einführenden Stichwortartikel von Lazlo A. Vascovics wird ein umfassender Überblick über die Ausdifferenzierung und Verbreitung der verschiedenen Formen sozialer Elternschaft mit Blick auf die Veränderung der Familien- und Verwandtschaftsformen ebenso gegeben wie über das spärliche Wissen über die Qualität dieser Eltern-Kind-Verhältnisse. Dabei wird zunächst der Begriff der sozialen Elternschaft in seinen verschiedenen Bedeutungsfacetten skizziert und in historischer Perspektive beleuchtet. Anschließend werden die differenten Strukturtypen und Formen von sozialer Elternschaft beschrieben, wobei zwischen klassischen Formen der sozialen Elternschaft wie etwa Stiefelternfamilien, Adoptiveltern, Pflegefamilien unterschieden wird, aber auch Formen nicht-familialer sozialer Elternschaft in stationären Einrichtungen der Kinder- und Jugendhilfe wie Heimen und Wohngruppen sowie neuere Formen sozialer Elternschaft, etwa in Gestalt von „Patchworkfamilien“ oder „Regenbogenfamilien“ einbezogen werden. Darüber hinaus wird auf der Basis empirischer Studien aufgezeigt, wie sich die verschiedenen Formen von Elternschaft im Lebenslauf verändern können und welche Konsequenzen diese Entwicklungen für den Wandel von Verwandtschaftssystemen von Kindern haben. Weiterhin wird auf der Grundlage quantitativer Befunde der aktuelle Verbreitungsgrad unterschiedlicher Formen sozialer Elternschaft in Deutschland skizziert und abschließend unter Berücksichtigung einer teilweise noch defizitären Forschungslage die Frage diskutiert, wie sich die Qualität von Elternschaft und das Erziehungsverhalten in Familien mit leiblichen Eltern von denen mit sozialen Eltern unterscheidet.

In dem Beitrag von Thomas Gabriel und Samuel Keller steht das Konzept der sozialen Elternschaft vor dem Hintergrund ausgewählter Befunde der ,Zürcher Adoptionsstudie“ im Mittelpunkt. Dabei werden zunächst die verschiedenen Dimensionen des Begriffs der sozialen Elternschaft erläutert und der nationale und internationale Forschungsstand zum Wandel hin zu multiplen Formen der Elternschaft und insbesondere zu den Folgen von Adoption für das Familienleben beschrieben. Anschließend stellen die Autoren zentrale Ergebnisse aus dem Kontext der Zürcher Adoptionsstudie vor, bei der in einer quantitativen und qualitativen Längsschnittstudie die Auswirkungen von Adoption auf die intersubjektiven Erfahrungen der Eltern untersucht wurden. Dabei wird verdeutlicht, wie gesellschaftliche Wandlungsprozesse, normative Ansprüche und familiale Leitbilder sowie behördliche Überprüfungen 
die Suche nach Normalität von Eltern beeinflussen und oft die eigenständige und sinnstiftende Entwicklung von ,sozialer Elternschaft“ erschweren.

Im Zentrum des Artikels von Christine Entleitner-Phleps und Sabine Walper steht die Analyse des kindlichen Wohlbefindens in Stiefelternfamilien und alleinerziehenden Familien im Vergleich zu Kernfamilien. Empirische Basis ihrer Untersuchung sind die Daten des repräsentativen DJI-Surveys „Aufwachsen in Deutschland: Alltagswelten", in dessen Kontext 2173 Kinder im Alter zwischen neun und zwölf Jahren im Jahr 2014 befragt wurden. Die Ergebnisse machen deutlich, dass Kinder, die in einer komplexen Stieffamilie oder mit einer alleinerziehenden Mutter leben, im Vergleich zu Kindern in Kernfamilien Nachtteile hinsichtlich des kindlichen Wohlbefindens zeigen, während dies für Kinder aus einfachen Stiefvaterfamilien nicht zutrifft.

Funding Open Access funding provided by Projekt DEAL.

Open Access Dieser Artikel wird unter der Creative Commons Namensnennung 4.0 International Lizenz veröffentlicht, welche die Nutzung, Vervielfältigung, Bearbeitung, Verbreitung und Wiedergabe in jeglichem Medium und Format erlaubt, sofern Sie den/die ursprünglichen Autor(en) und die Quelle ordnungsgemäß nennen, einen Link zur Creative Commons Lizenz beifügen und angeben, ob Änderungen vorgenommen wurden.

Die in diesem Artikel enthaltenen Bilder und sonstiges Drittmaterial unterliegen ebenfalls der genannten Creative Commons Lizenz, sofern sich aus der Abbildungslegende nichts anderes ergibt. Sofern das betreffende Material nicht unter der genannten Creative Commons Lizenz steht und die betreffende Handlung nicht nach gesetzlichen Vorschriften erlaubt ist, ist für die oben aufgeführten Weiterverwendungen des Materials die Einwilligung des jeweiligen Rechteinhabers einzuholen.

Weitere Details zur Lizenz entnehmen Sie bitte der Lizenzinformation auf http://creativecommons.org/ licenses/by/4.0/deed.de. 